\title{
Recent sheath physics studies on DIII-D*
}

\author{
J.G. Watkins ${ }^{1}$, B. Labombard ${ }^{2}$, P.C. Stangeby ${ }^{3}$, C.J. Lasnier ${ }^{4}$, A.G. McLean ${ }^{4}$, \\ R.E. Nygren ${ }^{1}$, J.A. Boedo ${ }^{5}$, A.W. Leonard ${ }^{6}$, and D.L. Rudakov ${ }^{5}$

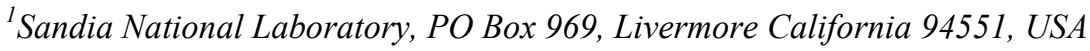 \\ ${ }^{2}$ MIT Plasma Science and Fusion Center, 175 Albany St, Cambridge, Massachusetts 02139, USA \\ ${ }^{3}$ University of Toronto Institute for Aerospace Studies, Toronto, M3H 5T6, Canada \\ ${ }^{4}$ Lawrence Livermore National Laboratory, 700 East Ave, Livermore, California 94550, USA \\ ${ }^{5}$ University of California San Diego, 9500 Gilman Dr., La Jolla, California 92093-0417, US \\ ${ }^{6}$ General Atomics, PO Box 85608, San Diego, California 92186-5608, USA
}

\begin{abstract}
A study to examine some current issues in the physics of the plasma sheath has been recently carried out in DIII-D low power Ohmic plasmas using both flush and domed Langmuir probes, divertor Thomson scattering (DTS), an infrared camera (IRTV), and a new calorimeter triple probe assembly mounted on the Divertor Materials Evaluation System (DiMES). The sheath power transmission factor was found to be consistent with the theoretically predicted value of $7(+/-2)$ for low power plasmas. Using this factor, the three heat flux profiles derived from the LP, DTS, and calorimeter diagnostic measurements agree. Comparison of flush and domed Langmuir probes and divertor Thomson scattering indicates that proper interpretation of flush probe data to get target plate density and temperature is feasible and could potentially yield accurate measurements of target plate conditions where the probes are located.
\end{abstract}

Abstract length (150 words): 139 currently 
PACS: 52.40.Kh, 52.40.Hf, 52.55.Fa, 52.55.Rk

PSI-20 keywords: Plasma Sheath, DIII-D, Divertor diagnostic, Langmuir probes, calorimeter *Corresponding author address: General Atomics, 3550 General Atomics Court, San Diego, CA 92121

*Corresponding author E-mail: watkins@fusion.gat.com 


\section{Introduction}

This paper examines two important physics issues involving the plasma sheath. The first issue concerns the heat flux to an object exposed to plasma. The resulting plasma sheath that forms has an electric field that accelerates ions into the plate. The sheath potential drop is determined by local plasma conditions. The incident heat flux is conveniently related to the nearby plasma conditions by the sheath power transmission factor (SPTF). This factor includes contributions to the heat flux from both the ion and electron populations. The SPTF is defined by the equation [1]: heat flux, $\mathrm{q}_{\|}=\gamma^{*} \Gamma^{*} \mathrm{k} \mathrm{T}_{\mathrm{e}}$, , where $\gamma$ is the SPTF, $\Gamma$ is the particle flux to the plate, and $\mathrm{kT}_{e}$ is the electron temperature. Discrepancies with this relationship near the divertor outer strike point have been previously found [2,3] where the particle flux and electron temperature from Langmuir probes were compared with the heat flux from an infrared camera (IRTV). In those experiments, the SPTF showed a large drop $(\gamma->1)$, below the theoretical minimum, near the strike point. Here, in this study, we measure the local heat flux with a $6 \mathrm{~mm}$ flush calorimeter probe [4] and the local target plate plasma conditions with a 6 mm domed Langmuir probe. The divertor Thomson scattering (DTS), located $0.8 \mathrm{~m}$ away along the magnetic field, provides density and temperature measurements to serve as a benchmark for comparison.

The second issue we examine here concerns the interpretation of measurements from flush Langmuir probes. Because flush Langmuir probes have less erosion, lower incident heat flux, and much longer operational lifetime, they are being considered for use in ITER. The interpretation of Langmuir probe data requires accurate knowledge of the

collection area. Because magnetic field lines generally have a small angle with the 
divertor plates, flush Langmuir probes usually have a much smaller projected area (along the magnetic field) and when the probe is biased, the collection area may increase significantly as the plasma sheath above it expands. Several theories [6-11] that describe the sheath expansion and the interpretation of flush probe data are well known. We have found success using one of these theories [9-11] to compare flush probe measurements with measurements of a nearby matching domed Langmuir probe and DTS.

\section{Experimental Setup}

ELM free Ohmic plasmas were used to provide more quiescent target plasma conditions for comparison of diagnostic measurements in the divertor. This study was conducted in DIII-D at a line-averaged density of $3.0 \times 10^{19} \mathrm{~m}^{-3}$, a plasma current of 1.07 MA, and a toroidal field of $-1.95 \mathrm{~T}$ with the grad $\mathrm{B}$ drift towards the $\mathrm{x}$-point. The outer divertor strike point radial position $\left(\mathrm{R}_{\mathrm{sep}}\right)$ was swept slowly $(6.7 \mathrm{~cm} / \mathrm{sec})$ across the fixed locations of the DIMES LPs and DTS to measure conditions at different points in the outer strike point plasma profiles.

The heat flux is measured with a calorimeter probe [4] by recording the temperature time history of a $6 \mathrm{~mm}$ diameter graphite collector flush with the DIMES sample surface. A $0.75 \mathrm{~mm}$ diameter thermocouple (TC) is spring loaded from below to measure the temperature $4 \mathrm{~mm}$ below the collector surface. The calorimeter collector is insulated from the tile using the same spring loaded insulating hardware as the domed Langmuir probes. The thermocouple is placed in the collector such that the temperature rise (at $4 \mathrm{~mm}$ ) is linear with time for a constant heat flux on the top surface. This configuration is sometimes called a lumped inertial mass. The analysis requires that magnetic pickup on the small TC voltage signal, mostly due to the toroidal field ramp up, is removed from the 
signal before conversion to temperature and then heat flux. The heat flux is derived from the slope of the temperature time history. Profiles of the heat flux can be obtained using slow sweeps of the strike point.

In order to make measurements along the same flux tube, the x-point location was adjusted to allow for alignment of the magnetic field lines between the DTS sample volumes and the DIMES sample containing the Langmuir probes and the calorimeter (shown in Fig. 1). Magnetic field line tracing was performed to confirm that alignment was achieved during the sweep. The magnetic configuration used for this alignment necessarily resulted in very shallow field line angles at the divertor plate (0.5-1.5 degrees) near the strike point.

The IRTV measures target plate surface temperature about 120 degrees $(9.4 \mathrm{~m})$ toroidally away from the DIMES location and heat flux is derived from the THEODOR 2D thermal model [5]. During this experiment, the IRTV was in line scan mode that measures the surface temperature profiles at about $10 \mathrm{kHz}$.

The spatial location of the Langmuir probes, the calorimeter, and the DTS were surveyed with a coordinate measuring machine $(\mathrm{CMM})$. This spatial survey occurred with the DIMES triple probe still in place. This spatial survey proved to be very useful for interpreting the results.

\section{Heat Flux measurements}

After correcting the calorimeter data for a small surface misalignment measured by the CMM survey, the heat flux measurements indicated that the calorimeter heat flux agreed (figure 2) with the heat flux determined from the other diagnostics using a SPTF of 7. A correction factor based on the ratio of the projected areas, $A_{\text {top }} / A_{\text {projected, was used }}$ 
to determine the perpendicular heat flux falling on the top surface of the calorimeter. The DTS heat flux is calculated using a sheath factor of 7 times the calculated particle flux (ne*Cs) times the measured electron temperature. Both the LP and DTS calculated heat fluxes includes the deposition of $15.8 \mathrm{eV}$ [12] of energy per particle gained from the ionization process and molecular breakup but this correction was less than 15 per cent in this case. All heat fluxes were converted to perpendicular heat flux by using the angle of incidence of the magnetic field lines with the surface from the magnetic equilibria calculated using EFITD65Y [13, 14].

Using the calorimeter heat flux and the domed LP particle flux and electron temperature (3-parameter fit), a profile of the SPTF was found (see figure 2). Near the strike point, the SPTF may be a little lower, reminiscent of previous experiments, but unfortunately many of the profile measurements were perturbed near the strike point by a reciprocating probe plunge during the strike point sweep. Combining data from multiple shots may help resolve the issue.

Heat flux profiles from the IRTV were corrupted by a hot divertor tile radial edge in the path of the line scan such that the normal heat flux profiles could not be used. IRTV heat flux profiles, were instead constructed from IRTV measurements at $R=1.49 \mathrm{~m}$ which were normalized to the strike point location during the sweep as was done with the other diagnostics (also at $\mathrm{R}=1.49 \mathrm{~m}$ ). The IRTV profiles constructed in this way did show strike point values of about $10 \mathrm{~W} / \mathrm{cm}^{2}$, similar to the other three heat flux profiles, but did not show much variation from that value over the outer parts of the profile. Either because of measurement surface alignment issues or lack of sensitivity at these low levels of heat flux, the IRTV heat flux profiles were not useful for determination of the SPTF. 
Except for the IRTV, the other three measurements were toroidally localized, especially the calorimeter and DIMES Langmuir probes, which were placed near each other by design for this experiment.

The peak in the radial heat flux profile, about $0.04 \mathrm{~m}$ into the scrape-off layer, is most likely due to changes in recycling when the poloidal flux surface angle was perpendicular to the horizontal target plate. There is a small difference between the three peak heat fluxes at $0.04 \mathrm{~m}$ and this is currently not explained but is within the measurement error. The lowest DTS channel R and Z location has been mapped into an effective target plate surface radial coordinate $\left(R_{\text {eff }}\right)$ to compare measurements on the same flux surface. $R_{\text {sep }}$ is the strike point radial coordinate on the top surface of the target plate.

\section{Flush probe measurements}

After correcting the flush probe projected area for a $0.07 \mathrm{~mm}$ top surface misalignment as was done for the calorimeter, the flush/domed current ratio was near the expected value and preliminary estimates, based on projected area calculations, indicated that the flush probe sheath expansion was 5-10 debye lengths above the surface. This is in the range of the theoretical predictions (Bergman factor $\left.=0.5 / \sin (\alpha)^{-0.5} \sim 5\right)[15,16]$ but, because the field line angles are less than three degrees, a more careful theoretical comparison will be needed.

To obtain $\mathrm{J}_{\mathrm{sat}}, \mathrm{T}_{\mathrm{e}}$, and density from the LPs, a four-parameter fit, including a term for sheath expansion with voltage, was used for I/V analysis of both the flush and domed probes (figure 3). It should be noted that all the profile data has been heavily smoothed for ease of comparison and, as mentioned earlier, the profiles from -0.005 to $0.01 \mathrm{~m}$ are perturbed due to a reciprocating probe plunge. The 3-parameter (solid line) fits are from 
fitting each voltage sweep while the 4-parameter (dashed line and triangles) fits were obtained from a $10 \mathrm{msec}$ average as described below. All fits used evenly weighted data for a more uniform comparison although normally the data is weighted more heavily near $V_{f}$ for the best $T_{e}$ fit. Although, the four-parameter fit does allow additional flexibility for a better $\mathrm{T}_{\mathrm{e}}$ match with DTS, it appears that the domed 4-parameter fit is more significantly perturbed near the strike point possibly due to the probe plunge or the "ground" current normally flowing there. To fit the sloping sheath expansion current, the fourth fit parameter was obtained from a linear fit of $\mathrm{I} \alpha \mathrm{V}^{3 / 4}$ in the ion saturation region of the I/V characteristic. The sloping part of the current (when shifted to intercept the origin) was subtracted away from the total current signal before fitting the corrected data with a three-parameter fit (shown in figure 4 as a dashed light blue curve). The usefulness of fitting this sheath expansion portion of the curve separately is described below.

Because of the noise levels from fluctuations, many voltage sweeps were combined together (10 msec, 5 complete sweeps, $10 \mathrm{~K}$ points) and binned along the voltage axis to get the median value in each of $\sim 60$ bins to use for the four-parameter fits. The $T_{e}$ derived from this 4-parameter analysis showed better agreement with the DTS temperature for both probes than the 3-parameter fit. The density analysis was done in several ways. Following the technique described by Gunn [9-11], the short circuit current $\left(\mathrm{V}_{\text {probe }}=0\right.$ intercept) was found by fitting the flush probe sheath expansion as a function of $\left(\mathrm{V}_{\text {plasma- }}\right.$ $\left.\mathrm{V}_{\text {probe }}\right)^{3 / 4}$. At $\mathrm{V}_{\text {probe }}=0$, the flush probe is at the target plate potential and the sheath is not expanded beyond the normal target plate sheath and, in this case, the normal projected area of the flush probe top surface can be used to get the density from $J_{\text {sat }}$ and $T_{e}$. The 
density profile extracted from the flush probe data using this short circuit technique is shown in Fig. 3 (triangles).

\section{Conclusions}

The measurement of the sheath power transmission factor, performed at low power but with plasma perturbations near the strike point, did agree with the typical sheath theory predictions. We also found that interpretation of flush Langmuir probe data with present theories yielded plasma profiles very similar to measurements made with divertor Thomson scattering and a nearby domed Langmuir probe. Preliminary estimates of the sheath expansion are within range of the theoretical predictions. Using the Gunn short circuit theory to interpret flush Langmuir probe data to get plasma density appears to work because the probe current is evaluated at the potential of the target plate $\left(\mathrm{V}_{\text {probe }}=0\right)$ where there is no bias and therefore no sheath expansion, which means that the projected area is the same as the effective collection area. Using probes with the same geometry as the target plate is less perturbing to the local recycling and will certainly have less erosion, lower intercepted heat flux, and longer probe tip lifetime than the "proud" or higher profile type of Langmuir probe tips. Although, this study is preliminary and needs more data with better surface alignment of the probes and fewer plasma perturbations to fully support these conclusions, it appears that the use of flush probes in ITER is feasible and could potentially yield accurate measurements of target plate conditions where the probes are located.

\section{Acknowledgment}

This work was supported in part by the US Department of Energy under DE-AC0494AL85000, DE-AC52-07NA27344, DE-SC0001961, and DE-FC02-04ER54698. 
DIII-D data shown in this paper can be obtained in digital format by following the links at https://fusion.gat.com/global/D3D_DMP.

\section{References}

[1] P.C. Stangeby, The Plasma Boundary of Magnetic Fusion Devices, IOP, Bristol, 2000

[2] A. Futch, et al, Jour. Nuc. Mater. 196-198 (1992) 860-864

[3] D. Buchenauer, et al, Rev. Sci. Instrum. 66 (1995) 827

[4] J.G. Watkins, et al, Rev. Sci. Instrum. 74 (2003) 1574

[5] A. Herrmann et al., Plasma Phys. Control. Fusion 37 (1995) 17

[6] M. Weinlich, A. Carlson, Phys. Plasmas 4 (1997) 2151

[7] U. Wolters, et al, Plasma Phys. Control. Fusion 41 (1999) 721 - 732

[8] T.H. Daube, et al. Contrib. Plasma Phys. 38 (1998) 145 - 150

[9] J. P. Gunn, et al, Rev. Sci. Instrum. 66 (1995) 154 - 159

[10] J. P. Gunn, et al, Contrib. Plasma Phys. 36 (1996) S 45 - 52

[11] J.P. Gunn, Phys. Plasmas 4 (1997) 4435 - 4446

[12] M. Wischmeier, "High density divertor operation for reactor-relevant power exhaust", to be published, Jour. Nuc. Mater. (2015)

[13] L. Lao, H. St. John, R.D. Stambaugh, and W. Pfeiffer, Nucl. Fusion 25, $1421(1985)$.

[14] L. Lao, et al, Nucl. Fusion 30, 1035 (1990).

[15] A. Bergman, Phys. Plasmas 1 (1994) 3598 - 3606

[16] A. Bergman, Phys. Plasma 9 (2002) 3413-3420 


\section{Figure Captions}

Fig. 1. This figure shows the domed and flush Langmuir probes and the flush calorimeter probe (lower picture - view from above) mounted on the DIMES sample. The side view (upper diagram) shows how the DTS scattering volumes are oriented vertically above the target plate at a different toroidal location. The solid horizontal line shown in the side view indicates the height of the DIMES sample surface and Langmuir probes. The calorimeter and domed probes are toroidally aligned at the same radial position. The dashed vertical line indicates where $\mathrm{R}=1.49 \mathrm{~m}$.

Fig. 2. This figure shows the comparison of three heat flux radial profiles from the domed Langmuir probe, the calorimeter probe, and divertor Thomson scattering. The DTS and Langmuir probe heat fluxes are obtained using a sheath factor of 7 . The measured sheath factor shown is calculated from the domed probe and calorimeter data and agrees with the theoretically predicted value within 30 per cent. $\mathrm{R}_{\mathrm{eff}}$ is the projected radial location of the DTS lowest channel along the magnetic field onto the target plate surface. For the probe and calorimeter data, $\mathrm{R}_{\mathrm{eff}}=\mathrm{R}_{\text {probe }}$.

Fig. 3. Profiles of particle flux $\left(\mathrm{J}_{\mathrm{sat}}\right)$, electron temperature $\left(\mathrm{T}_{\mathrm{e}}\right)$, and density are shown from the domed Langmuir probe (dashed -4 parameter fit, and solid -3 parameter fit, solid), the flush probe (triangles), and the lowest DTS channel (boxes - DTS @ $7.6 \mathrm{~mm}$ above the plate). The flux and temperature shown for the flush probe were determined from the four parameter fit using $10 \mathrm{msec}$ data averaging and the flush probe projected area and ion current corrected for the surface misalignment. The domed 3-parameter fit here does not include the sheath expansion as was done for the domed 4-parameter fit but does fit every voltage sweep individually. The flush probe density (triangles) was 
determined from the I0 "short circuit" model $(9,10,11)$ after doing a four parameter fit (Vaxis intercept $=$ flush-I0).

Fig. 4. This figure shows a comparison of flush (upper solid red curve) and domed (lower dashed red curve) I/V characteristics using a 4-parameter fit. The 3-parameter fit done after removing the sloped part of the $\mathrm{I}_{\text {sat }}$ current is also shown for the domed probe (dashed cyan fit). The short circuit current ( $\mathrm{I} 0$ ) at $\mathrm{V}_{\text {probe }}=0$ is found by linearly extrapolating (grey straight line) the sloped region of the "saturated" part of the I/V curve for both the domed and flush characteristics. The slope in the $\mathrm{J}_{\mathrm{sat}}$ indicates sheath expansion. The short circuit current is actually found by extrapolating a linear fit of $\mathrm{I}_{\text {probe }}$ verses $\mathrm{V}_{\text {probe }}{ }^{3 / 4}$ to the vertical $\mathrm{V}_{\text {probe }}=0$ axis. This is the $\mathrm{I} 0$ current used to calculate the density from the projected area in figure 3 . The vertical dashed green line shows the floating potential $\left(V_{f}\right)$ from the fits. A slight difference is sometimes seen in $V_{f}$ for the two 4-parameter fits. 


\section{List of Figures}

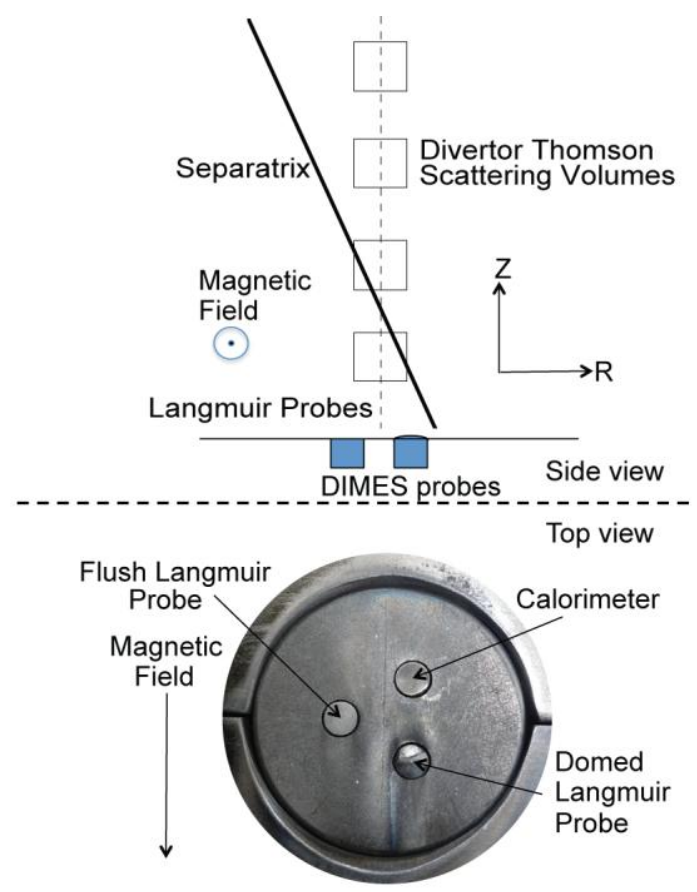

Fig. 1 


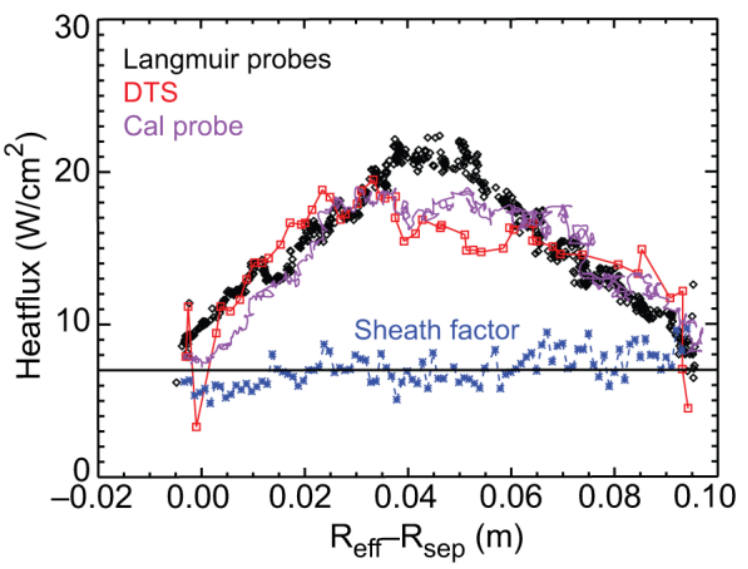

Fig. 2 

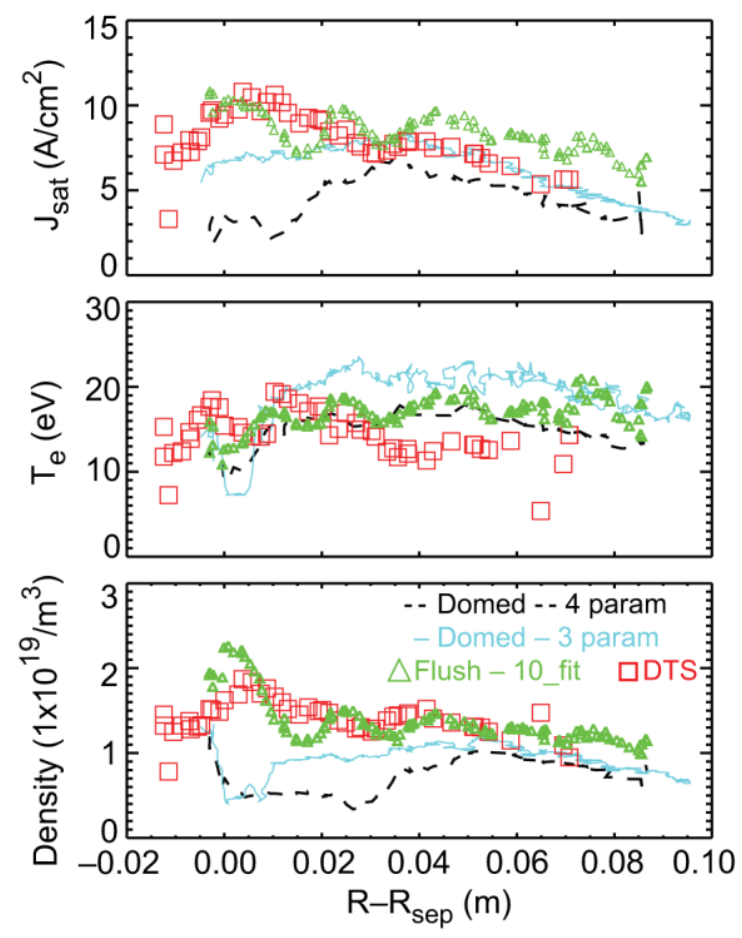

Fig. 3 


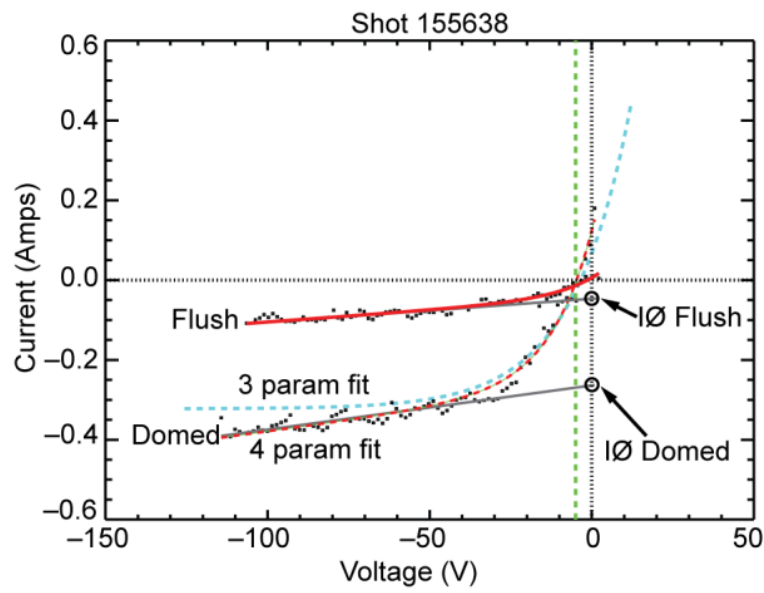

Fig. 4 


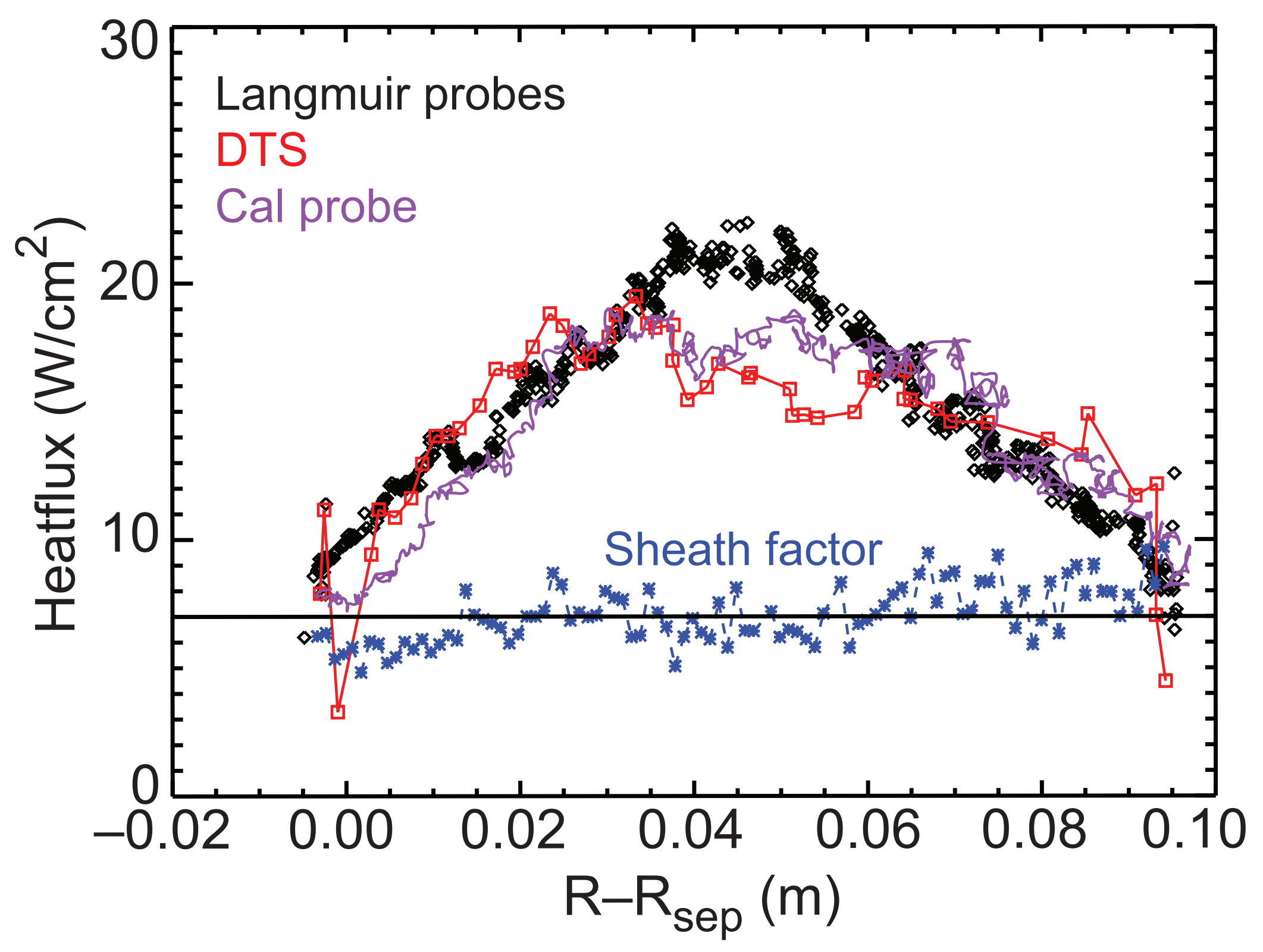


Figure
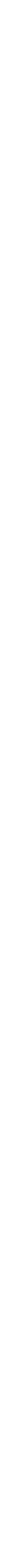


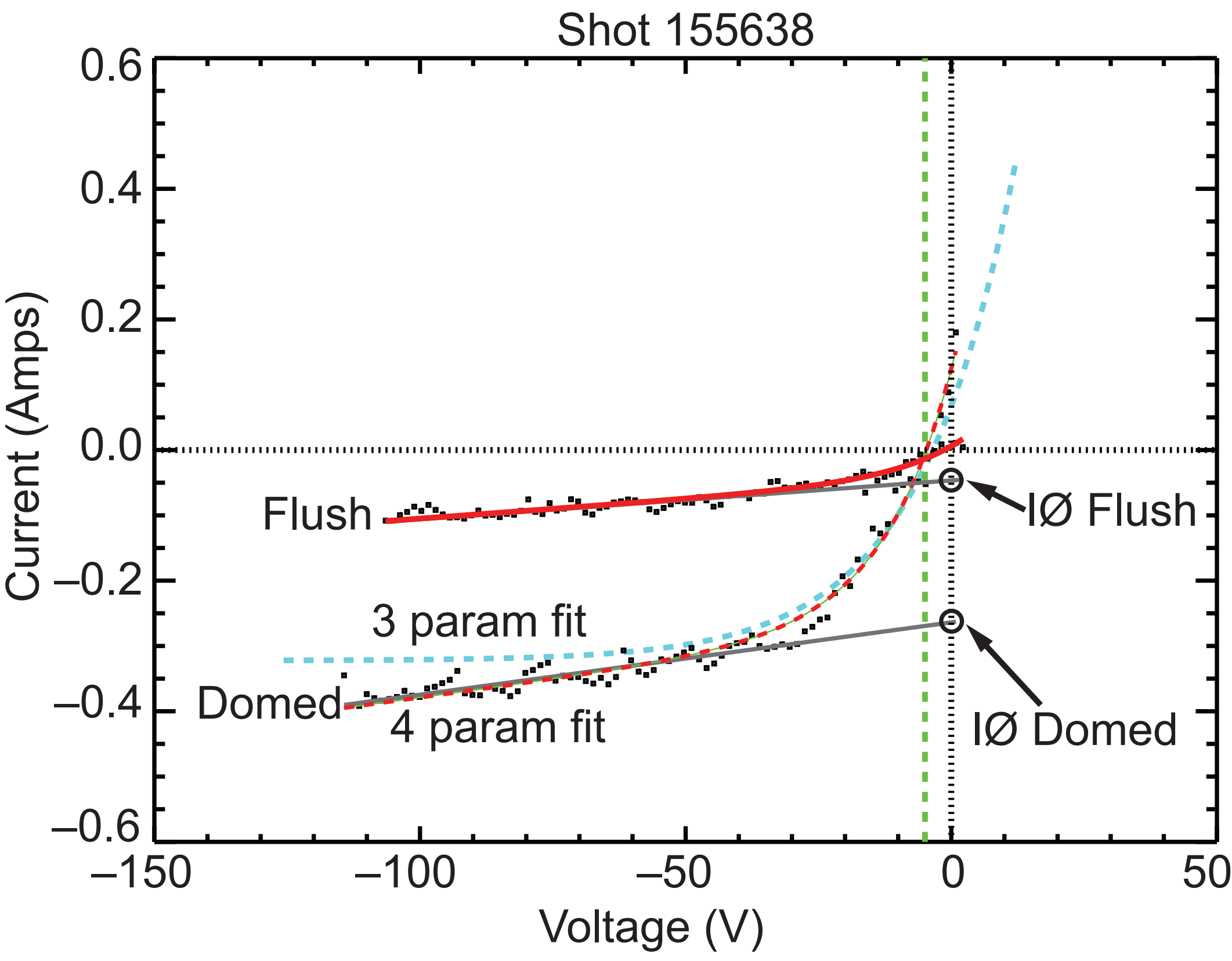

